\title{
Image Compression and Reconstruction using DOST and DCST
}

\author{
${ }^{* 1}$ Padigala Prasanth Babu, ${ }^{2}$ T. Jayachandra Prasad, ${ }^{3}$ K. Soundararajan \\ ${ }^{1}$ Dept of ECE, JNTUA, Ananthapuramu, Andhra Pradesh, India. \\ ${ }^{2}$ Dept of ECE, RGMCET, Nandyala, Andhra Pradesh, India. \\ ${ }^{3}$ Dept of ECE, TKR College of Engineering, Hyderabad, Telangana, India. \\ Email: prasanthbabu.padigala@gmail.com, talari.jp@gmail.com, soundararajan_jntucea@yahoo.com
}

Received: 17th April 2020, Accepted: 05th May 2020, Published: 30th June 2020

\begin{abstract}
Image compression is a key step in the processing of images to reduce the data size and speed of image transmission and storage. Wavelets are usually said to be the innovative technique to compress the image, but the output of waves on smooth parts of the image is suboptimal. A new technique for image compression using Stockwell Transform is therefore being proposed. In this work MATLAB technical computing language is used to implement the proposed Stockwell Transforms. The quality parameters such as Mean Square Error, Peak Noise Ratio, Normalized Cross-correlation, Average Difference, Content Structural, Maximum Difference and NAE are computed to assess the methods.
\end{abstract}

Keywords

Stockwell Transform, DOST, DCST, Compression, Orthonormal.

\section{Introduction}

The art and science of reducing the amount of data needed for an image is image compression in the field of digital image processing, which is the most helpful and commercially successful technologies [1]. The compression algorithms and specifications benefit from the use of a digital camera, videos and web surfing. According to the principles of television engineering, the video is translated into images. A two-hour Standard Definition TV movie needs $224 \mathrm{~GB}$ of data storage, where a total of 27 out of $8.5 \mathrm{~GB}$ of dual-layer DVDs are provided for data storage.

The data should be compressed by a factor of 26.3 for storage on a single disk and the compression rate for highdefinition TV would increase. In order to reduce storage space and increase the speed of transmission image compression, web pictures and high-resolution digital camera pictures are also used. A residential internet connection provides data for broadband with a speed of 56 to $12 \mathrm{Mbps}$. So, the time needed to transmit an image of $128 \times 128 \times 24$ bit color over the speed range is 7.0 to 0.03 seconds. When the image is compressed, the time of transmission can be decreased by a factor of 2 to 10 and more.

In further fields such as video conference, remote sensing, facsimile transmission (FAX), document and medical imaging image compression plays an important role. Image compression offers growing applications, depending on how effectively the binary, gray scale and color images are treated, processed and transmitted.

Today, with the growth of new commuting technologies, image compression needs are growing rapidly. Image compression is the only solution to the problem of digital image delivery and the storage of large numbers of digital image files. Compression decreases the storage space by maintaining the image quality. Image compression covers applications such as television broadcasting, satellite remote sensing and other long-range communication. There are two methods of compression-lossy compression and lossless compression. Lossless does not require any data loss during compression and reconstruction, while data loss occurs in loss compression. Lossy methods are used in applications for signal processing. Image compression can be accomplished by using Discrete Cosine Transform, where the transform is a sum of cosine functions, which are oscillated at different magnitudes and frequencies. A 2D Discrete Cosine Transform is used and implemented using simple JPEG hardware, but when the compression ratio is high,'blocking objects' cannot be ignored. But the image quality restored degrades by true contouring.

In the Discrete Wavelet Transform (DWT) of an image, the sum of wavelet functions known as wavelets and the size and position of wavelets are different. Discrete wavelet transformation (DWT) represents images as a set of High and Low pass data, first dividing the image into $32 \times 32$ blocks and then crossing two filters for each block. A transformation matrix is generated and passed through the desired compression by a constant scaling factor. Daubuchies are typically used for compression of images. On smooth image sections, discrete wavelet transformation (DWT) output is suboptimal. The use of DOST and DCST for image compression has been explored as an alternative to this traditional approach. This paper presents a comparison of DOST and DCST.

\section{Related Work}

Yanwei wang, et.al [2] have presented and investigated the discrete orthogonal stockwell transform (DOST) for image compression. This transform provides a multiresolution spatial- frequency representation, higher value of PSNR and better quality of the image. In the smooth regions of 2D image compression, the distribution of errors 
suggests that this transform technique outperformed the wavelet compression techniques.

The Discrete Rajan Tranforms (DRT) have been presented and discussed for higher quality compression [3-4]. The image compression using DRT is compared and analyzed with the different wavelet transforms. The performance of DRT is evaluated in terms of image quality metrics such as Mean Square Error, Peak Signal to Noise Ratio, Normalized Cross Correlation, Average Difference, Structural Content, Maximum Difference and Normalized Absolute Error. The simulation results of DRT are better than DCT and DWT.

\section{Stockwell Transforms}

The Wavelet Transform (WT) is good for extracting time and frequency data fields and is noise sensitive [7]. The STFT has a fixed resolution and phase information is not included in the Wavelet Transform. This led to the development of a new transform known as the Stockwell Transform, an extension of the Wavelet Transformation or STFT variable window [13]. It is based on the scalable Gaussian localization window and provides frequency dependent resolution [6]. The $\mathrm{S}$ transform is known for its local spectral phase characteristics. The key characteristic of the S-Transformations is that they uniquely combine a frequency-dependent time-frequency resolution with referenced local phase information [2].

$\mathrm{S}$ Transform provides the frequency-based resolution through direct control of the Fourier spectrum relationship. The wavelet transform does not satisfy the zero mean condition so it is not a Continuous Wavelet transformation. The $\mathrm{S}$ transform is extremely stable in noise conditions and can effectively define and process non-stable signals [2].

S-Transforms could be used in both medical and non-medical applications [5]. It is used in geophysics, gravitational wave detection, image transmission/compression, and so on in non-medical applications [9-10]. The following approach is followed by considering standard images to test the output of the S Transform (DOST and DCST) for image compression. The sequence of steps involved in the algorithm is as given below:

1. Read the Standard input Image.

2. Compute the DOST/DCST coefficients of the Image.

3. Compute the Compression Ratio by applying Run Length Coding.

4. Compute the inverse DOST/DCST for the compressed image reconstruction of original image

5. Calculate the Image Quality Measurement parameters

The block diagram of Image Compression and Decompression using DOST and DCST is shown in the figure.1.

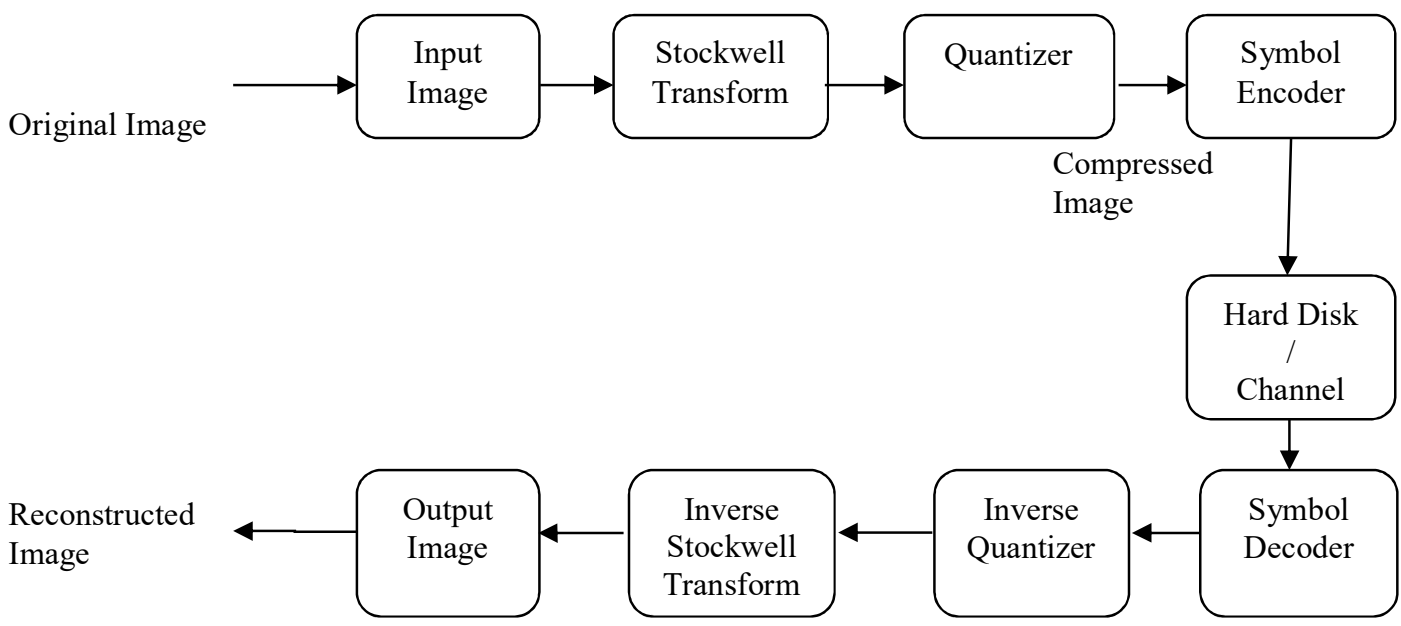

Fig.1: The Block Diagram of Image Compression and Decompression using DOST and DCST.

\section{Image Quality Measurement Parameters}

This section makes a breakthrough on the consistency calculation parameters used to equate DOST and DCST for Discrete Orthonormal Stockwell Transform. The digital images are subject to a number of distortions which can be created, processed, compressed and stored. Distortions that reduce image quality may also occur during transmission and reconstruction [11]. Mean Squared Error (MSE) and Peak Signal to Noise Ratio (PSNR) are generally used Image Quality metrics [8]. MSE and PSNR require simple calculations and have a distinct meaning, which is logical and not well suited for visual quality [12]. The quality of reconstructed image can also be assessed by the following quality measurement parameters [4].

\section{A. Mean-squared Error (MSE)}

The equation (1) is the mean squared error for a two-dimensional image of a scale $(\mathrm{MxN})$

$\mathrm{MSE}=\frac{1}{\mathrm{MN}} \sum_{\mathrm{x}=1}^{\mathrm{M}} \sum_{\mathrm{y}=1}^{\mathrm{N}}\left[\mathrm{I}(\mathrm{x}, \mathrm{y})-\mathrm{I}^{\prime}(\mathrm{x}, \mathrm{y})\right]^{2}$ 
Here $\mathrm{I}(\mathrm{x}, \mathrm{y})$ is the input image (or) original Image and I'(x, y) is the output image or reconstructed image. If the MSE value is lower, it implies that there is less number of errors in the reconstructed image.

B. Peak Signal to Noise Ratio (PSNR)

The PSNR is the measure of peak error in the reconstructed image and is given by equation.(2)

$\mathrm{PSNR}=10 \log _{10}\left(\frac{255^{2}}{M S E}\right)$

From the equations (1) and (2), it can be seen that there is an inverse relation between PSNR and MSE. The lower the MSE value, the higher the PSNR. Higher PSNR value means that the signal-to-noise ratio is higher.

C. Normalized Cross-Correlation (NCC)

A commonly used parameter which helps to determine the degree of similarity or difference between the image original and reconstructed. The NCC value normally varies from -1 to 1 . The image alignment in the time series is accurate if the value of the NCC is ' 1 ' but the amplitude can vary and it is represented in equation (3).

$\mathrm{NCC}=\sum_{x=1}^{M} \sum_{y=1}^{N} I(x, y) \cdot I^{\prime}(x, y)$

\section{Average Difference (AD)}

Average Difference is defined as $\mathrm{AD}=$ Error $/(\mathrm{MxN})$, Error $=$ (Original Image - Reconstructed Image $)$

$A D=\frac{1}{M N} \sum_{x=1}^{M} \sum_{y=1}^{N}\left[I(x, y)-I^{\prime}(x, y)\right]$

\section{E. Structural Content (SC)}

Structural contents decide the degree of correspondence in pictures. The more common regions of both the original pictures and the restored ones, the more similar they are. The equation for Structural Content is given by equation.(5)

$\mathrm{SC}=\frac{\sum_{x=1}^{M} \sum_{y=1}^{N}[I(x, y)]^{2}}{\sum_{x=1}^{M} \sum_{y=1}^{N}\left[I^{\prime}(x, y)\right]^{2}}$

\section{F. Maximum Difference (MD)}

It gives the variation of the method of paired comparisons. It is given by equation.(6)

$\operatorname{MD}=\operatorname{Max}\left(\left|I(x, y)-I^{\prime}(x, y)\right|\right)$

\section{G. Normalized Absolute Error (NAE)}

This gives the numerical difference between the original and the reconstructed image given by equation.(7)

$\mathrm{NAE}=\frac{\sum_{x=1}^{M} \sum_{y=1}^{N}\left|I(x, y)-I^{\prime}(x, y)\right|}{\sum_{x=1}^{M} \sum_{y=1}^{N} I(x, y)}$

\section{Results and Discussion}

In this work, the Lena image as shown in Fig. 2 is considered for experimental investigation and is subject to both DOST and DCST methods. These methods help to compress the image through the transformation of the corresponding algorithms and produce the reconstructed image of the original image as shown in Figures 2 and 3.
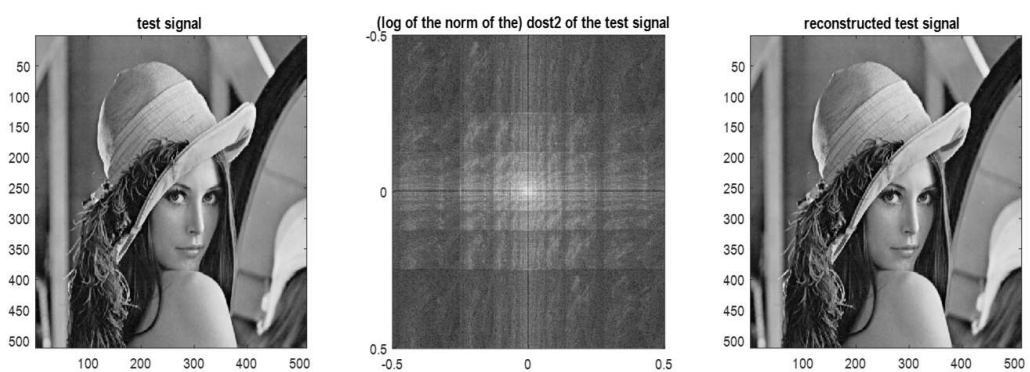

Fig.2: Original, Compressed and Reconstructed Image Using DOST
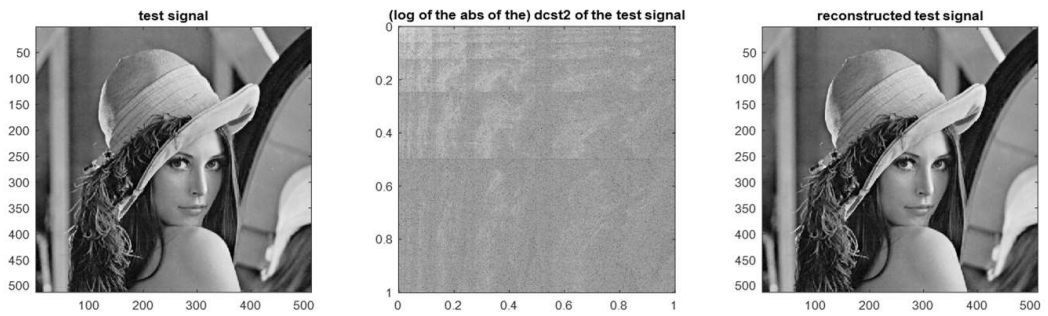

Fig. 3: Original, Compressed and Reconstructed Image Using DCST 
These methods are further investigated on various images which are mentioned in Table. 1 The accuracy of the reconstructed image will be assessed using the standard measurement criteria, namely MSE, PSNR, NCC, AD, SC, MD and NAE. 1. MSE is, for example, a very simple and very common measure of distortion. The MSE value is the difference between the processed image and the test image. The smaller the MSE, the better the outcome. PSNR is also one of the most commonly used metric parameters for the calculation of reconstruction efficiency.

A higher PSNR value indicates a higher quality reconstruction. The structural quality is a global metric that compares the overall weight of the picture compressed to the original. The structural container with a weight of 1, shows a better image quality. Average low value disparity suggests a good image quality. The normalized correlation gives the original picture closeness to the decode. NAE, which is a measure to study the approximation quality of the maximum value images. It is clear that statistical DCST metrics are closer to the ideal values and that significantly better results are achieved.

Table 1: Results of Various Test Images for DOST and DCST

\begin{tabular}{|c|c|c|c|c|c|c|c|c|}
\hline & MSE & PSNR & $\mathrm{NCC}$ & $\mathrm{AD}$ & $\mathrm{SC}$ & MD & NAE \\
\hline \multicolumn{2}{|c|}{ Ideal Values } & 0 & $\alpha$ & 1 & 0 & 1 & 0 & 0 \\
\hline \multirow{2}{*}{$\begin{array}{l}\text { Lena } \\
\text { Image }\end{array}$} & DOST & 603.103 & 20.3247 & 0.9654 & 1.2623 & 1.0337 & 223 & 0.0866 \\
\hline & DCST & 220.3886 & 24.6989 & 0.9945 & 0.0719 & 0.9973 & 167 & 0.0665 \\
\hline \multirow{2}{*}{$\begin{array}{l}\text { Barbara } \\
\text { Image }\end{array}$} & DOST & 1488.9 & 16.4021 & 0.9688 & $\begin{array}{l}-0.0788 \\
\end{array}$ & 0.9914 & 238 & 0.1669 \\
\hline & DCST & 2130.2 & 14.8466 & 0.9405 & -0.0732 & 1.0188 & 248 & 0.2206 \\
\hline \multirow{2}{*}{$\begin{array}{l}\text { Moon } \\
\text { Image }\end{array}$} & DOST & 531.9047 & 20.8725 & 0.9926 & -3.5249 & 0.9582 & 119 & 0.1207 \\
\hline & DCST & 494.8005 & 21.1865 & 0.9635 & -2.3952 & 1.0194 & 225 & 0.1687 \\
\hline \multirow{2}{*}{$\begin{array}{l}\text { Camerm an } \\
\text { Image }\end{array}$} & DOST & 5761.2 & 10.5257 & 0.7775 & 6.082 & 1.412 & 255 & 0.4068 \\
\hline & DCST & 6374.9 & 10.0861 & 0.7201 & 11.2131 & 1.6397 & 255 & 0.4873 \\
\hline \multirow{2}{*}{$\begin{array}{l}\text { Joker } \\
\text { Image }\end{array}$} & DOST & 5821.7 & 10.4803 & 0.8061 & 19.6978 & 1.361 & 254 & 0.3032 \\
\hline & DCST & 4076.3 & 12.0281 & 0.7675 & 30.0638 & 1.6092 & 255 & 0.3094 \\
\hline \multirow{2}{*}{$\begin{array}{l}\text { Marble } \\
\text { Image }\end{array}$} & DOST & 574.7151 & 20.5363 & 1.0014 & -11.0185 & 0.9114 & 147 & 0.3111 \\
\hline & DCST & 1454 & 16.5051 & 1.107 & -26.1512 & 0.6891 & 252 & 0.5538 \\
\hline
\end{tabular}

\section{Conclusion}

The removal of noise from unwanted images is a significant and difficult problem in the era of image enhancement and computer vision. It is therefore of great importance to restore the damaged picture to its simple state. This paper offers a comparative analysis of different DOST and DCST image quality metrics. Different parameters were checked for different images from different data sets based on two images. Each metric has a role to play in monitoring the quality of the image. It is thus found that DCST method in quantitative as well as parametric analysis has exceeded DOST.

\section{References}

1. G. K. Wallace,: The JPEG Still Picture Compression Standard, Source Communication of ACM, vol. 34, pp. 30-44, (1991).

2. Y. Wang., J. Orchard.: On the use of the Stockwell transform for image compression, Proc. SPIE 7245 Image Processing: Algorithms and Systems VII, pp. 7245-724504, (2009).

3. S. Sridhar, P. Rajesh Kumar, K.V. Ramanaiah,: Wavelet Transform Techniques for Image Compression - An Evaluation, I.J. Image Graphics and Signal Processing 2014, vol. 2, pp. 54-67, (2014).

4. Kethepalli Mallikarjuna, Kodati Sathya Prasad, Makam Venkata Subramanyam,: Image Compression and Reconstruction using Discrete Rajan Transform based Spectral Sparsing, I.J. Image Graphics and Signal Processing 2016, vol. 1, pp. 59-67, (2016).

5. Yusof, Y. W. Mohamad, Saparon.A.: Performance Comparison of Discrete Orthonormal S-Transform for the Reconstruction of Medical Images. Paper presented at the Modelling Symposium (EMS), pp.128-132,IEEE European (2015).

6. Stockwell RG: Why use the S-transform. Volume 52. A Wong. Fields Institute Communications, Providence, pp.279-309. Pseudo-Differential Operators: PDEs and Time-Frequency Analysis, (2007).

7. Neelamma K.Patil, V.R.Udupi, Vasudha.S.: S-Transforms - A Review, International Journal of Electrical, Electronics and Computer Systems, vol. 1, pp. 33-37, (2013).

8. Christopoulos C, Skodras A, Ebrahimi T : The JPEG2000 still image coding system: an overview,Consumer Electronics, IEEE transactions, Volume 46, pp.1103-1127, (2000).

9. J. Ladan, Edward R. Vrscay,:The Discrete Orthonormal Stockwell Transform and Variations, with Applications to Image Compression Image Analysis and Recognition, Lecture Notes in Computer Science, Volume 7950, pp 235-244, (2013). 
10. Y. Wang,: Efficient Stockwell transform with applications to image processing, PhD thesis, University of Waterloo, Ontario Canada, (2011).

11.D.S.Taubman , M.W.Marcellin,: JPEG2000: Image compression fundamentals, standards and practice, Kluwer Academic Publishers, (2002).

12. Poobal, S., Ravindran, G.: The performance of fractal image compression on different imaging modalities using objective quality measures. In: Proceedings of the International Journal of Science and Engineering Technology (IJEST 2011), vol. 3(1), pp. 525-530 (2011).

13. Lin Yun, $\mathrm{Xu}$ Xiaochun, Li Bin, Pang Jinegeng,: Time Frequency Analysis based on the S-Transform, International Journal of Signal Processing, Image Processing and Pattern Recognition, vol.6, no.5, pp 245254, (2013). 